\title{
Structural Localization of Some Phosphatases in the Golgi Region of Cultivated Cells
}

\author{
Hideo Masuda ${ }^{1}$ \\ Department of Biology, University of Osaka Prefecture, Sakai, Japan
}

Received January 28, 1967

\section{Introduction}

A number of studies have been attempted to elucidate chemical substances or enzymes specifically localized in a kind of cell organelles, but most of them have failed to prove the Golgi bodies. However, localization of thiamine pyrophosphatase and acid and alkaline phosphatase activities in the Golgi region was suggested by some investigators. Recently, Novikoff and Goldfischer (1961) discovered that thiamine pyrophosphatase activity was localized specifically in the Golgi apparatus of various types of cells, especially concentrated in the lamellae components of the Golgi complex. Their results were confirmed by Allen and Slater (1961) and Allen (1961), who demonstrated the enzyme activity in the corresponding fraction in electrophoretic studies, and by other investigators (Otero-Vilardebó, Lane and Godman 1964, Lazarus and Wallace 1964, Robbins and Gonatas 1964). These findings suggested that the enzyme activity might be available as a cytochemical technique for identification of the Golgi apparatus in the cultivated cells.

The Golgi region in the living cultivated cells is seen under the phase microscope as a prominent area applied to one side of the nucleus, which consists of intertwined phase-gray structures and phase-leucent vacuoles embedded in a ground substance (Takagi and Masuda 1956, Rose 1961, Masuda 1965, 1967). As reported previously, silver grains were not found at the coat of the phase-lucent vacuoles in these cells after silver impregnation according to Da Fano's or Elftman's method (Masuda 1967). On the other hand, the phase-gray constituents in the Golgi region observable in the living condition were preferentially impregnated by silver and transformed into silvered figures similar to those constituting the Golgi apparatus in routine preparations (Masuda 1967). In this sense, the present author logically speculated that the phase-gray constitutents are the true representatives of the Golgi apparatus demonstrable by classical metallic impregnations. However, there still remains some doubt about identifying the phase-gray constituents as the Golgi bodies, because the silver grains often percipitate on other cytoplasmic inclusions under various conditions during the process of impregnation. From this viewpoint, it is thought worth while to investigate precisely where the thiamine pyrophosphatase activity is localized.

Alkaline phosphatase activity was found in the Golgi region of intestinal epithelial cells (Novikoff, Korson and Spater 1952, Fredricsson 1956) and cultivated cells (Henrichsen 1956). Also, acid phosphatase activity in the Golgi region of cultivated cells was demonstrated by many investigators (Maggi and Riddle 1965, Jervis and Labrec 1966, Adachi and Pollak 1965). In the present study, an attempt was made to investigate the sites of activities of the three kinds of enzymes in the cell, especially in the Golgi region, using cultivated cells which can be precisely examined with phase optics in the living condition. The structures that contain the enzyme activities were compared in their topography and morphology with those of the Golgi region in the living cell.

\section{Material and method}

Tissue culture: Explants, which were excised from the meninges of chick embryos incubated

1 Present addres: Department of Biology, Kōnan University, Kobe, Japan. 


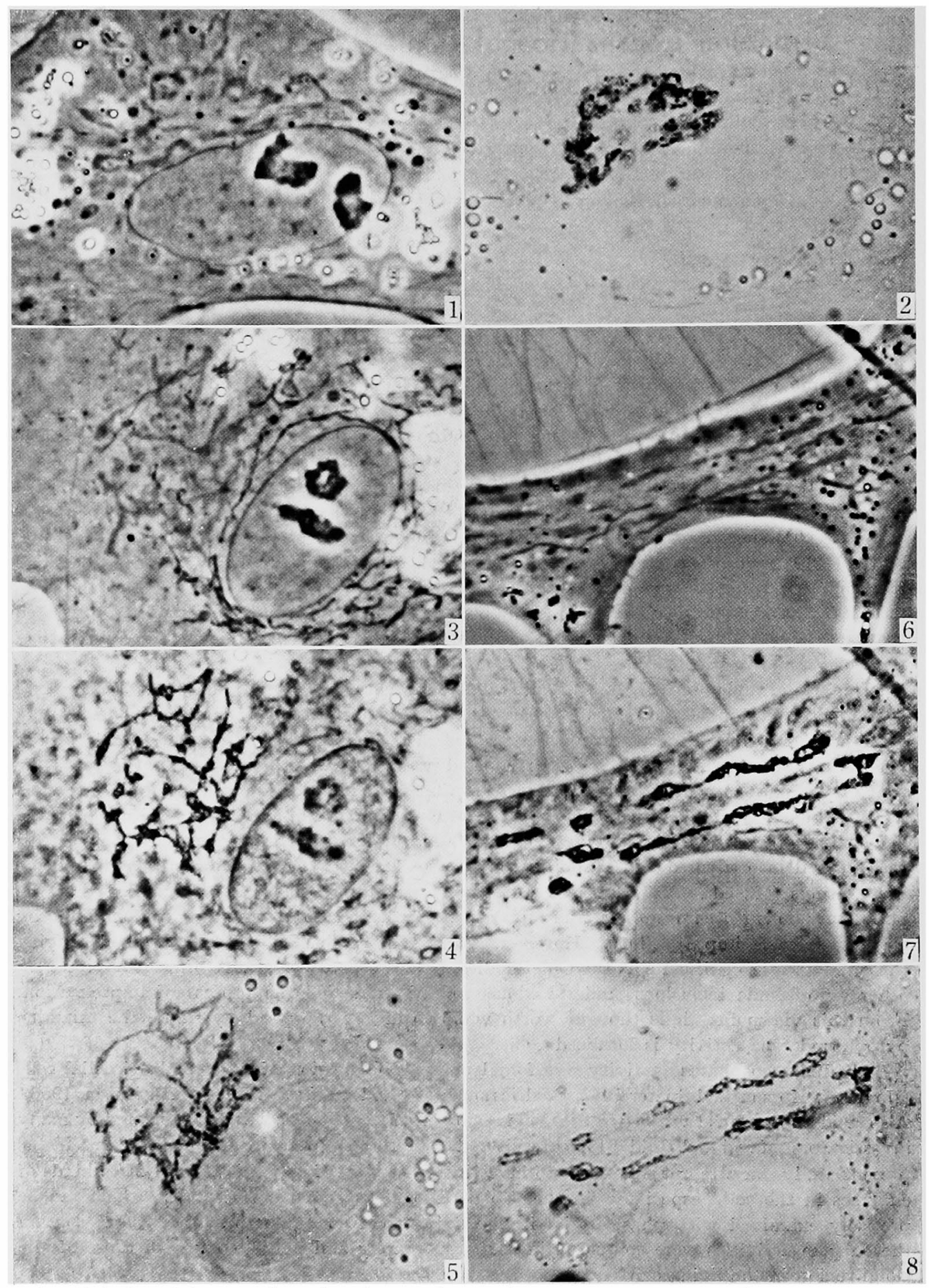

Figs. 1-8. 1 and 2, a portion of a living cell revealed through the phase microscope and the same cell after staining for thiamine pyrophosphatase (TPPase) activity. 1 shows the most common form of the accumulation of the phase-gray flecks, most of them being located in the peripheral part of the Golgi region. 2( to the right of Fig. 1) indicates the TPPase activity that is entirely cofinded to the phase-gray flecks. While the grains of the reaction products 
for 10 days, were cultured in multipurpose culture chambers developed by Rose and his coworkers (1958) and completely covered with a full sheet of cellophane just in the same manner as in the previous works. Among the cells that migrated out from the explants, those with the Golgi region containing sharply-delineated phase-gray constituents were selected and used as materials throughout this investigation. The living cells were carefully observed and recorded in photomicrographs under the phase microscope equipped with a NDH (Neo Dark High) objective (Tiyoda Optical Co.). The observation and photographing were immediately followed by placing the cells into fixatives to avoid morphological alterations. They were then subjected to one of the following tests of enzymes.

The test for thiamine pyrophosphatase: Materials were fixed overnight in a cold $4 \%$ formol-calcium solution. After rinsing in cold distilled water, some of the materials were incubated in thiamine pyrophosphate medium dissolved in Tris-maleate buffer solution (pH 7.2) at $37^{\circ} \mathrm{C}$ for 30 to 50 minutes as introduced by Novikoff and Goldfischer (1961). But most of them were incubated in a substrate medium modified by Naidoo (1962). Since rather weak deposition was obtained with a $10 \mathrm{mM}$ solution of thiamine pyrophosphate, its concentration was increased threefolds as recommended by Novikoff and Goldfischer (1961). For following in steps the deposition of reaction products on the cytoplasmic inclusions, incubation in the substrate medium was made to last for $15,30,45,60$ and 80 minutes. Control cells were incubated in the substrate medium containing uranyl acetate as an inhibitor.

The test for alkaline phosphatase: To demonstrate the alkaline phosphatase activity, materials were incubated in the alkaline sodium-glycerophosphate medium and precipitates were converted to visible products as described by Gomori ('52).

The test for acid phosphatase: Materials were fixed overnight in a cold $4 \%$ formolcalcium solution and fixation of some preparations was also attempted in a cold $6 \%$ formolcalcium solution for 1.5 hours. After rinsing in cold distilled water, coverslips were incubated in the presence of sodium-glycerophosphate in accordance with the method of Gomori (1950). As the control, some materials were tested by the same technique but with the addition of sodium fluoride to the substrate medium.

The localization of the enzyme activities demonstrated by these methods was carefully compared with the cytoplasmic inclusions in photomicrographs of the same cells taken in the living condition.

\section{Result}

Observation of the Golgi region in the living cells. As was described in my previous paper, the Golgi region is clearly recognized as a paranculear zone in most of the living cells under the phase microscope. In this area, many phase-gray constituents are embedded in a phase-lucent ground substance, which does not differ in contrast from the other cytoplasmic portions (Figs. 1 and 2). They are distinguished from the mitochondria, which are often intricated among them,

encrust the flecks in the peripheral part of the Golgi region, they are scarcely found on the flecks in the innermost part, 3,4 and 5 , a part of a cell illustrating phase-gray flecks to form a network to the upper left of the nucleus. 3, a photomicrograph taken through the phase microscope in living condition. Many fine phase-gray constituents can be seen intertwined with each other in the Golgi region. Following the test for TPPase activity (Fig. 4: under the phase microscope, Fig. 5: under the ordinary light microscope), the reaction products are deposited at the same loci with and in a configuration entirely similar to the phase-gray constituents in the living cell in Fig. 3.6, a phase microscope photograph illustrating a portion of an elongated cell in living condition, with the phase-gray flecks arranged in two rows parallel to the longer axis of the cell. 7 (taken through the phase microscope) and 8 (through the ordinary light microscope) indicate the TPPase activity in the same cell to that of Fig. 6 , which is entirely confined to the phase-gray flecks.

All figures (Figs. 1-16) are part of the cells showing the Golgi region at a magnification of 1700 times. 

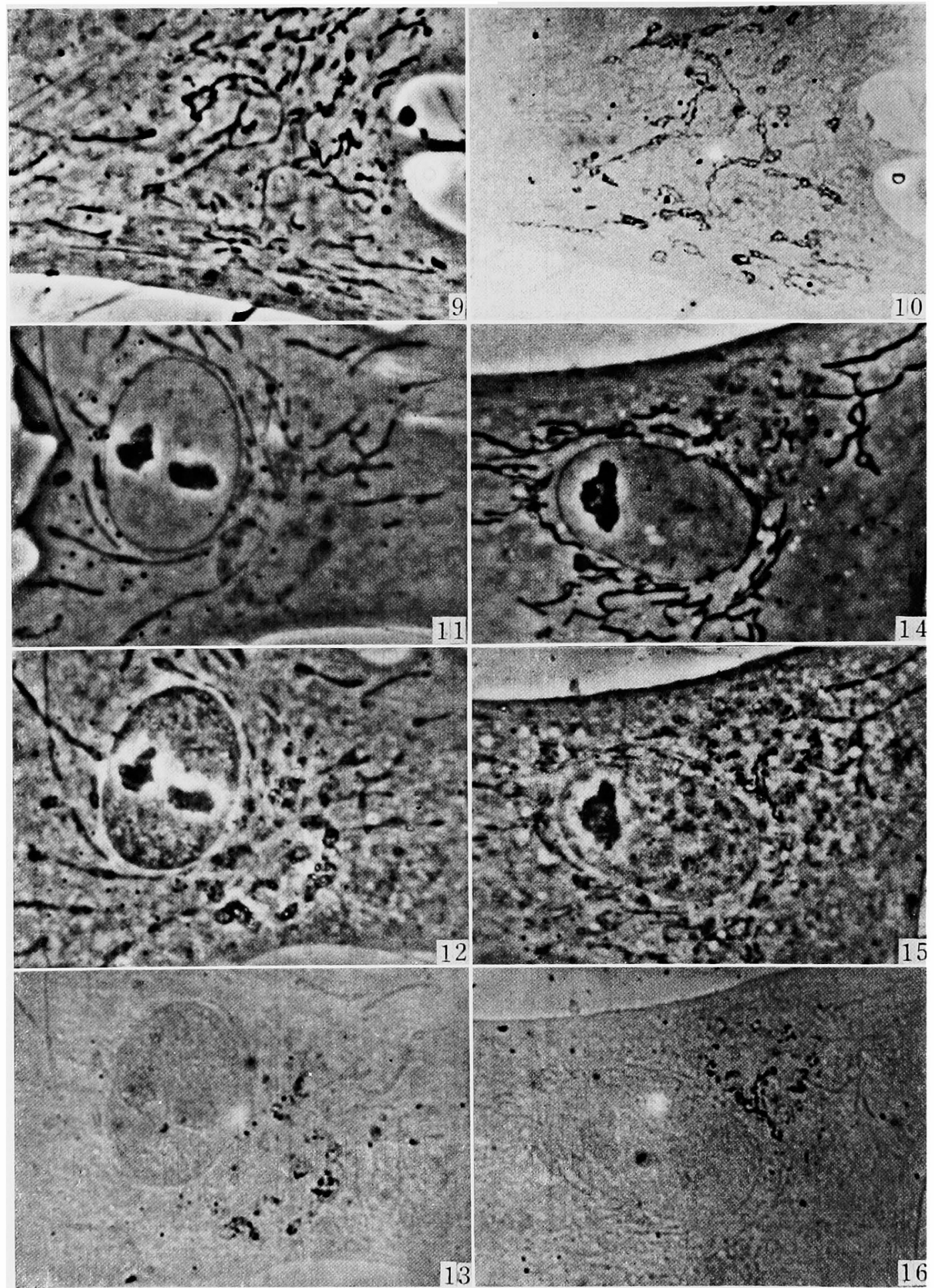

Figs. 9-16. A part of a well-spread cell in the living condition and after the test for TPPase activity. 9 the phase-gray flecks are found scattering disorderly among the mitochondria and removed far from the nucleus. 10 (to the right of Fig. 9) shows the TPPase 
without difficulty on account of their lesser contrast under the phase microscope. The phase-gray structures are generally filament- or rod-like in shape and show distinct but sometimes blurred outlines. They appear to be altered in their arrangement, shape and contrast in varying environmental conditions. Sometimes, a long phase-gray filament is seen to be fragmented into short ones under the microscope. The phase-gray flecks with ill-defined outline are sometimes very difficult to recognize, because they are not much different from the surrounding cytoplasm in contrast. On the contrary, sharply delineated phase-gray flecks are favorable materials for studying their morphological details and following their changes in location under the phase microscope. In the well-spread cells, the flecks are found very often to be widely scattered among the mitochondria (Figs. 9 and 10).

The phase-gray flecks usually appear as rod-shaped fragments. They are gathered in a cluster in the Golgi region, or most of them are situated in the peripheral part and the remainders are scattered disorderly in the more central part of that region (Figs. 1 and 2). They often lose their sharply delineated contour line during the course of observation and photographing, as if they were being dissolved into the surrounding cytoplasm, and finally come to be hardly recognizable. In the elongated cells, long filamentous phase-gray constituents are found very often lying among the mitochondria (Figs. 6, 7 and 8).

In some cells, several phase-lucent vacuoles ranging from 0.3 to $2 \mu$ in diameter are observed among the phase-gray flecks (Fig. 6). They are usually moving to and fro in the Golgi region and are often found far from the original sites.

Thiamine pyrophosphatase activity. With fixation overnight in a cold $4 \%$ formol-calcium solution, the phase-gray flecks turn darker and can be more sharply defined in outline. Hence, their shapes and positions are clearly observed under the phase microscope. Morphological comparison between the phase-gray flecks in the Golgi region of the living cells found by phase microscope and the reaction products provided with the enzyme activity leaves no doubt that the latter occur in the cell impregnating solely the former or in close association with them. In other words, the reaction products are entirely similar to the phase-

activity in the same cell. The grains of reaction products are deposited on the lateral surfaces of the phase-gray flecks leaving a white core and thus simulating a canalicular structure as has been observed in the case of silver impregnation of the Golgi bodies. 11, a phase microscope photograph of a portion of a living cell showing an accumulation of the phase-gray flecks to the lower right of the nucleus. 12 and 13 indicate the acid phosphatase activity in the Golgi region of the same cell shown in Fig. 11. By dint of phase optics, the grains of reaction products can easily be recognized lying on the phase-gray flecks. 14, a part of a cell containing an accumulation of many small phase-gray flecks to the upper right of the nucleus and phase-white vacuoles scattering in and around the Golgi region as revealed by phase microscope. 15 and 16 indicate the acid phosphatase activity in the same cell shown in Fig. 14. The grains of the reaction products are localized on the surfaces of the flecks to form a canalicular structures as in the test for TPPase activity. It is obviously seen that the phase-white vacuoles do not present any enzymatic activity. 
gray constituents in their morphology and topography. In cells incubated for a short duration in the substrate medium, minute grains are visualized on the surfaces of the phase-gray flecks (Figs. 3, 4 and 5). On the larger flecks, the grains are observed to be attached on each lateral surface leaving a white core in just the same way as the silver grains in the course of metallic impregnation (Figs. 9 and 10).

As the incubation in the substrate medium goes on, the reaction products gradually grow in size, and even in a material incubated for a longer period, almost all grains can be distinguished from the neighboring ones. Though the reaction products finally cover the phase-gray flecks to form the canalicular structures very similar to those of the classical Golgi apparatus demonstrated by impregnation with heavy metals, each of the flecks visualized by the reaction products looks more slender and delicate in general than the fragments of the classical Golgi apparatus (Figs. $6,7,8,9$ and 10). Sometimes, the deposition of reaction products takes place in a different manner, i.e. even between the phase-gray flecks in the Golgi region. It also occurs that in the Golgi region, which contains the phase-gray flecks gathered either compactly or loosely in a cluster, the peripherally located flecks are completely encrusted by the reaction products, while some centrally located ones remain without any staining (Figs. 1 and 2).

The activity of thiamine pyrophosphatase is not found at any other cytoplasmic inclusions nor in the ground substance; that is, the only phase-gray structures in the Golgi region are enzymatically active in the cultivated cells.

Acid phosphatase activity. Although the cultivated cells that have been treated overnight in a cold $4 \%$ formol-calcium solution show scarcely the enzymatic activity, those fixed in a cold $4 \%$ or $6 \%$ formol-calcium solution for 1 to 3 hours exhibit considerable deposition of the reaction products in the Golgi region. Furthermore, precise observation reveals that the reaction products are almost entirely confined to the phase-gray constituents in the Golgi region and nuclear and perinuclear staining does not occur in any of the cells. After 30 minutes incubation in the substrate medium, a minute quantity of reaction products appear on the surfaces of the phase-gray flecks. They are smaller in number in most flecks than those produced by the thiamine pyrophosphatase activity. They are scattered at intervals on the surfaces of the phase-gray flecks and usually do not come in contact with neighboring ones (Figs. 11, 12 and 13). Further, they increase gradually in size independently of each other as the incubation proceeds, so that the phase-gray flecks visualized by the reaction products are not seen so slender as in the test of thiamine pyrophosphatase activity. In some cell, the phase-gray constituents are almost completely surrounded by the reaction products and become an appearance obtained by the procedure of silver impregnation (Figs. 14, 15 and 16). Acid phosphatase activity cannot be observed in any other part of the Golgi region including the phase-white spherules, so-called secretory droplets of Rose (1961).

No visible staining occured in all materials incubated for 2 to 8 hours in the 
substrate medium for alkaline phosphatase activity.

\section{Discussion}

From the exmaination of thiamine pyrophosphatase activity under the microscope, it is evident that the reaction products first appear as minute grains on the lateral surfaces of the phase-gray constitutents observable in the living cells. As incubation in the substrate medium goes on, the grains grow in size and come in contact mostly with their neighbours on the same surface area. Finally, they completely encrust the phase-gray flecks leaving a white core or center. The manner in which the reaction products come to be associated with the surface of the phase-gray flecks is supririsingly similar to that in impregnation by the Elftman's direct silver method (1952). However, the grains of the reaction products are not so numerous as the silver grains in metallic impregnation and seem to grow in their size independently from the others, thus resulting in much larger grains. They usually cover each of the phase-gray flecks with a smooth contour line. However, prolonged incubation in the substrate medium causes the phase-gray flacks to become surrounded by reaction products with a rugged and irregular outline, as was the case in materials heavily impregnated with silver. Therefore, the phase-gray flecks visualzied by the demonstration of the enzyme activity are somewhat different in shape from the same in the living condition. However, they appear to resemble those in the living cell more closely in their morphology than those demonstrated by silver impregnation.

Novikoff and Goldfischer (1961) discovered that the thiamine pyrophosphate was hydrolzyed specifically at the system of the double membranes of the Golgi complex. As seen in the text figures, the grains of the reaction products are associated only with the surfaces of the phase-gray flecks observed in the living cultivated cells and none of the enzyme activity is found on any other cytoplasmic inclusions. In some cells of the snails, phospholipid granules were reported to contain the enzyme activity in their cortical portion (Lee 1963, Lane 1963, 1964, Meek and Bradbury 1963, Meek and Lane 1964). Therefore, the fact that no deposition of the reaction products occurs on the oil droplets sometimes produced in the cultivated cells suggests that these droplets are composed of triglycerides.

Frequently, reaction products are found scattered on the surfaces of cells and even on the surfaces of coverslips outside the cells, especially when incubated for a longer period in the substrate medium. The grains here are comparatively larger than those lying on the phase-gray flecks. Naidoo (1962) also reported such grains deposited on the cellular and nuclear membranes in neurones of the thiamine deficient rats. In the present study, photomicrographic comparison of the same cells taken before and after staining indicated that no organelles can be recognzied at the location in the living cells where the scattered grains are found after the staining. These circumstances lead to conclude that the scattered grains are not suggestive of any thiamine pyrophosphatase activity of the cells, and that the 
enzyme activity is confined to, or almost exclusively concentrated on, the surfaces of the phase-gray flecks in the Golgi region observable in the living condition.

In the previous paper, the present author reported a conclusion that the phasegray constitutents are equivalent to the components of the living Golgi complex based on the evidences obtained by the silver impregnation method, which has universally been accepted as a standard method for demonstrating the Golgi apparatus. However, it has often been alluded to that the silver impregnation techniques for the Golgi apparatus reveal not only the cytoplasmic organelle, but also fat droplets, mitochondria or other inculsions according to a variety of conditions. This fact raised a question as to whether the phase-gray flecks exactly corresponded to the components of the Golgi apparatus.

Recently, the test for thiamine pyrophosphatase activity has proved to be extremely useful in demonstrating and identifying the Golgi lamellae (Novikoff and Goldfischer 1961, Allen 1963). The result of the present study further supports the belief that the phase-gray constituents in the Golgi region of the living cultivated cells are equivalent to the double membrane system of the Golgi complex; a conclusion reached by the previous study in the use of Elftman's silver direct method.

It was previously reported that the phase-gray flecks which are located more centrally in the Golgi region are hardly impregnated with silver, while those at the periphery are easily encrusted with silver grains. Similar figures of distribution are also obtained with grains in the demonstration of the thiamine pyrophosphatase activity. It may be due to the fact that the substrate is hydrolzyed mostly at the peripheral part, and supplied in a trifling amount to the center of the Golgi region. But, the possibility is not excluded that a variety of functional activities are not equally distributed among the phase-gray flecks in the Golgi region. It may be a future problem to solve the question whether or not individual phase-gray flecks in the Golgi region exhibit different physiological activities.

Many authors investigated the acid phosphatase activity in cultivated cells and revealed that it was concentrated in the Golgi region which was the thickest part of the cells (Fortelius, Levonen and Saxén 1961, Maggi and Riddle 1965, Adachi and Pollak 1965). This region of the cells cultivated in ordinary methods is usually filled with mitochondria, fat droplets and numerous minute vacuoles, most of the last being supposedly lysosomes. These cytoplasmic inclusions interfere considerably with detailed observation of the morphological and topographical features of the phase-gray constitutents. Hence, it could not be established which of the cytoplasmic inclusions contains the acid phosphatase activity in the Golgi region of the cells. It may be supposed that the most part of the activity is localzied in the lysosomes in the Golgi region. On the other hand, the cells cultivated in the multipurpose culture chamber completely covered with dialysis cellophane comprise only a few, if any, phase-bright granules in the Golgi region. In these cells, it is able to study the fine structure of this region under the phase microscope. As shown in the text figures, acid phosphatase activities are 
localized in the phasegray constitutents and the reaction products are found on their surfaces, a white core being left just as in the case of Elftman's impregnation method and of deposition of reaction products in the test for thiamine pyrophosphatase activity. Namely, the reaction products are seemingly almost entirely confined to each lateral surface of the stack of lamellae of the Golgi complex.

It is well known fact that the acid phosphatase activity is concentrated in the vacuoles which are assumed to be lysosomes and that a staining of the enzyme activity is widely used as lysosomal marker. The reaction products in the Golgi region are usually found in the vacuoles near the lamellae under the electron microscope. Recently, intense activity of the acid phosphatase was found in the saccules of the Golgi complex as well as in the delimiting membrane being considered to belong to early secretion vacuoles in pancreatic B-cell of rabbits (Lazarus and Volk 1965, Lazarus, Volk and Barden 1966) and jejunal epithelial cells of mouse under the electron microscope (Barka 1964). The biochemical studies of the Golgi fraction obtained from epididymis have also indicated the presence of the activity of acid phosphatase in the Golgi apparatus (Kuff and Dalton 1959). In regard to the lamellae, the findings obtained in this work about acid phosphatase are in agreement with the results of the above authors and, besides, give another support to the proposition that the phase-gray flecks correspond to the lamellae component of the Golgi complex.

On the basis of the observations, Novikoff and his collaborators have proposed that some of the lysosomes arise from the Golgi vacuoles which are formed by dilation of the membrane system in secretory cells, nuerones and hepatoma cells (Novikoff, Essner and Quintana 1964, Goldfischer, Essner and Novikoff 1954). In the cultivated cells, the phase-white vacuoles were already suggested to be likely emanated from the phase-gray flecks (Rose 1961. Masuda 1967). Nevertheless, the phase-white vacuoles observed in the living cells do not present any of the acid phosphatase activity as shown in the figures (Figs. 14, 15 and 16). Such observations seem to suggest that the phase-white vacuoles in the cultivated cells are not the representatives of the lysosomes observable under the electron microscope, or that the lamellae system of the Golgi complex can produce the vacuoles with different functional activities according to different physiological conditions of the cells.

The present author wishes to express his hearty thanks to Prof. S. Takagi, Shukugawa-gakuin Junior College, for his valuable advices in the course of the present study.

\section{Summary}

1. Localization of some phosphatase activities was investigated in the cells cultivated in the multipurpose culture chambers developed by Rose.

2. The activity of thiamine pyrophosphatase was specifically confined to the surface of the phase-gray flecks in the Golgi region. The reaction products encrusted the phase-gray flecks and formed what looked like a tubule with 
chromophilic walls and a chromophobic lumen, which was similar to a Golgi body demonstrated by metallic impregnation in vertebrate somatic cells.

3. Based on theevidences obtained in the study, it was justified that the phase-gray flecks were the true representatives of the Golgi apparatus in the living cultivated cells and supposedly correspond to the double membrane systems of the Golgi complex observed under the electron microscope.

4. The activity of the acid phosphatase was also found to be associated with the phase-gray flecks, but not with the phase-white vacuoles in the Golgi region which were presumably considered to be lysosomes or secretory droplets.

5. In many preparations showing thiamine pyrophosphatase and acid phosphatase activities, while deposits of the reaction products were found in a very small quantity on the phase-gray flecks in the more central porition of the Golgi region, they were concentrated in a greater quantity on the more peripherally located flecks in the same region. No significant staining was obtained in the test for alkaline phosphatase.

\section{Literature cited}

Adachi, M. and Pollak, O.J. 1965. Rabbits corneal cells studied in tissue cultures. II Enzyme reactions. Z. Zellfrosch. 70: 284-289.

Allen, J.M. 1961. A cytochemical study of Golgi associated thiamine pyrophosphatase in the epididymis of the mouse. J. Histochem. Cytochem. 9: 629-630.

- and Slater, J.J. 1961. A cytochemical study of Golgi associated thiamine pyrophosphatase in the epididymis of the mouse. J. Histochem. Cytochem. 9: 418424 .

- 1963. The properties of Golgi associated nucleoside diphosphatase and thiamine pyrophosphatase. I Cytochemical analysis. J. Histochem. Cytochem. 11: 529-541.

Barka, T. 1964. Electron histochemical localization of acid phosphatase activity in the small intestine of mouse. J. Histochem. Cytochem. 12: 229-238.

Elftman, H. 1952. A direct silver method for the Golgi apparatus. Stain Tech. 27: 47-52.

Fredricsson, B. 1956. Alkaline phosphtase in the Golgi substance of intestinal epithelium. Exp. Cell Res. 10: 61-65.

Fortelius, P., Levonen, E. and Saxén, E. 1961. Cytochemical demonstration of some enzyme system in HeLa cells. Acta Path. Microbiol. Scand. 52: 23-32.

Gomo:i, G. 1950. An improved histochemical technic for acid phosphatase. Stain Tech. 25: $81-85$.

- 1952. Microscopic Histochemistry., The University of Chicago Press.

Goldfischer, S., Essner, E. and Novikoff, A.B. 1964. The localization of phosphatase activity at the level of ultrastructure. J. Histochem. Cytochem. 12: 72-96.

Henrichsen, E. 1965. Alkaline phosphatase in osteoblasts and fibroblasts cultivated in vitro. Exp. Cell Res. 11: 115-127.

Lazarus, S.S. and Volk, B.W. 1965. Ultrastructure and acid phosphate distribution in the pancreas of rabbits. Arch. Path. 80: 135-147.

-, Volk, B.W. and Barden, H. 1966. Localization of acid phosphatase activity and secretion mechanism in rabbit pancreatic B-cells. J. Histochem. Cytochem. 14: 233-246.

- and Wallace, B.J. 1964. Nucleoside phosphatase and thiamine pyrophosphatase activity of rabbit Golgi apparatus. J. Histchem. Cytochem. 12: 729-736.

Jervis, H.R. and Labrec, E.H. 1966. Correlation of acid phosphatase activity with degree of bacterial infection in HeLa cells. J. Histochem. Cytochem. 14: 196-202.

Kuff, E.L. and Dalton, A.J. 1959. in "Subcellular Particles" edited by T. Hayashi. 
Lane, N.J. 1963. Thiamine pyrophosphatase, acid phosphatase and alkaline phosphatase in the neurone of Helix aspersa. Quart. J. Micr. Sci. 104: 401-12.

Lane, N.J. 1964. Localization of enzymes in certain secretory cells of Helix tentacles. Quart. J. Micr. Sci. 105: 49-60.

Lee, R.S. 1963. Phosphatase activity in the neurones of Locusta migratoria. Quart. J. Micr. Sci. 104: $475-481$.

Maggi, V. and Riddle, P.N. 1965. Histochemistry of tissue culture cells: A study of the effect of freezing and some fixatives. J. Histochem. Cytochem. 13: 310-317.

Masuda, H. 1965. On the Golgi apparatus of the cultivated cells before and after impregnation with silver. Bull. Univ. Osaka Pref. Ser. B 16: 1-11.

- 1966. On the phase-gray structures in the Golgi region of the living cultivated cells. Cytologia 32: 42-54.

Meek, G.A. and Bradbury, S. 1964. Localization of thiamine pyrophosphatase activity in the Golgi apparatus of a mollsusc, Helix aspersa. J. Cell Biol. 18:73-85.

- and Lane, N.J. 1964. The ultrastructural localization of phosphatase in the neurones of the snail, Helix apsersa. J. Roy. Micr. Soc. 82: 193-204.

Naidoo, D. 1962. Thiamine diphosphatase: a histochemical study. J. Histochem. Cytochem. 10: $580-591$.

Novikoff, A.B. and Goldfischer, S. 1961. Nucleosidediphosphatase activity in the Golgi apparatus and its usefulness for cytological studies. Proc. Nat. Acad. Sci. 47: 802810.

- Essner, E. and Quintana, N. 1964. Golgi apparatus and lysosomes. Federation Proc. 23: $1010-1022$.

- Kroson, L. and Spater, H.W. 1952. Alkaline phosphatase activity in the Golgi substance of intestinal mucosa. Exp. Cell Res. 3: 617-618.

Otero-Vilardebó, R., Lane, N. and Godman, G.C. 1964. Localization of phosphatase activities in colonic goblet and absorptive cells. J. Cell Biol. 21 : 486-490.

Robbins, E. and Gonatas, N.K. 1964. The ultrastructure of a mammalian cell during the mitotic cycle. J. Cell Biol. 21 : 429-464.

Rose, G.G., Pomerat, C.M., Schindler, T.O. and Trunnell, J.B. 1958. A cellophane-strip technique for culturing tissue in multipurpose culture chambers. J. Biophysic. Biochem. Cytol. 4: 761-764.

- 1961. The Golgi complex in living osteoblasts. J. Biophysic. Biochem. Cytol. 9: 463-478.

Takagi, S. and Masuda, H. 1956. Fine structure of the Golgi apparatus of the fibroblasts in vitro as revealed by phase microscopy and vital staining. Bull. Univ. Osaka Pref. Ser. B. 6: 33-41. 